\title{
Cyclic voltammetric method with stripping of the metal deposit for quantitative analysis of organic additives in metal plating electrolytes
}

\author{
L.N. Solodkova* and V.E. Kasatkin \\ A.N. Frumkin Institute of Physical Chemistry and Electrochemistry, Russian Academy of \\ Sciences, Leninsky pr. 31, 119071 Moscow, Russian Federation \\ *E-mail: Ins42@bk.ru
}

\begin{abstract}
Cyclic voltammetic method with stripping (CVS) is used to determine the concentration of organic additives of various natures in electrolytes for metal coatings used for the manufacture of printed circuit boards (PCB). It is based on the quantitative determination of the inhibiting or activating effect of the additive depending on the concentration in the bath. Since the rate of metal deposition in the CVS cathodic cycle depends on the content of the additive in the electrolyte, the charge spent on stripping metal in the anodic cycle is proportional to the additive concentration and can be used as an analytical signal. To increase the stability of the results, measurements are carried out in a special cell with a rotating micro disk electrode, ensuring the constancy of hydrodynamic conditions at the electrode. The previously patented "Korian" method developed on basis CVS is adapted to the IPC brand potentiostats by means of a specialized application for a personal computer in addition to the standard program of the serial IPC device. This program allows you to fully automate the analysis of additives by controlling the operating modes of the potentiostat and processing the results obtained. The analysis results are presented in digital form and graphically. The principles of realization of the analysis of the various nature organic additives in various electrolytes (not only PCB) by the CVS method "Korian" are discussed. Specific examples show the possibilities of quantitative determination of the concentration of organic additives in industrial baths, their efficiency, consumption and stability during storage and electrolytic deposition of metal coatings. This method and the "Korian" hardware and software complex is used at many plants in Russia and abroad.
\end{abstract}

Keywords: plating electrolytes, analysis, organic additive, cyclic voltammetry, korian.

Received: October 2, 2020. Published: November 25, 2020

doi: $\underline{10.17675 / 2305-6894-2020-9-4-19}$

\section{Introduction}

To improve the structure and functional properties of electrolytic deposits, various organic additives and their combinations are usually introduced into electrolytes. The concentration of such additives, as a rule, is not high and changes due to oxidation and reduction at the electrodes, also by adsorption and/or inclusion into the electrolytic deposit. These changes in the composition of the electrolyte affect the structure and properties of metal coatings, 
and, therefore, require quantitative control. It should be noted that the additives used are products of the synthesis of a number of organic compounds of various nature. In this regard, the main disadvantage of known control methods (optical spectroscopy, chronoamperemetry, chromatography, electrochemical impedance spectroscopy, stripping voltammetry, etc.) is the lack of universality in the determination of organic substances of various natures [1]. In addition, using these methods, without additional research, it is impossible to separate the fraction of the influence of the organic additive active component from its decomposition products and from impurities that do not affect (or, conversely, have a negative effect) on the structure and properties of metal deposits. In industrial practice, a simple qualitative method is mainly used to control the appearance of a galvanic deposit obtained in a special Hull cell [2]. This method makes it possible to estimate the integral effect of additives and impurities on the properties and quality of the coating, without giving information about their composition and, most importantly, about concentration [3].

The mechanism of the organic additives action on the structure and properties of metal deposits is based on the adsorption-diffusion model [4], according to which the adsorption of organic matter on growing metal crystals affects the rate of its deposition. On the basis of these concepts, the "Korian" method was developed for the quantitative determination of organic additives in the electrolytes, as well as the estimation of the effectiveness of singleand multicomponent additives action on the structure and properties of the deposited metals $[5,6]$. The analysis is based on the methods of linear-speed cyclic and pulse voltammetry with stripping of the metal deposit (CVS and PCVS).

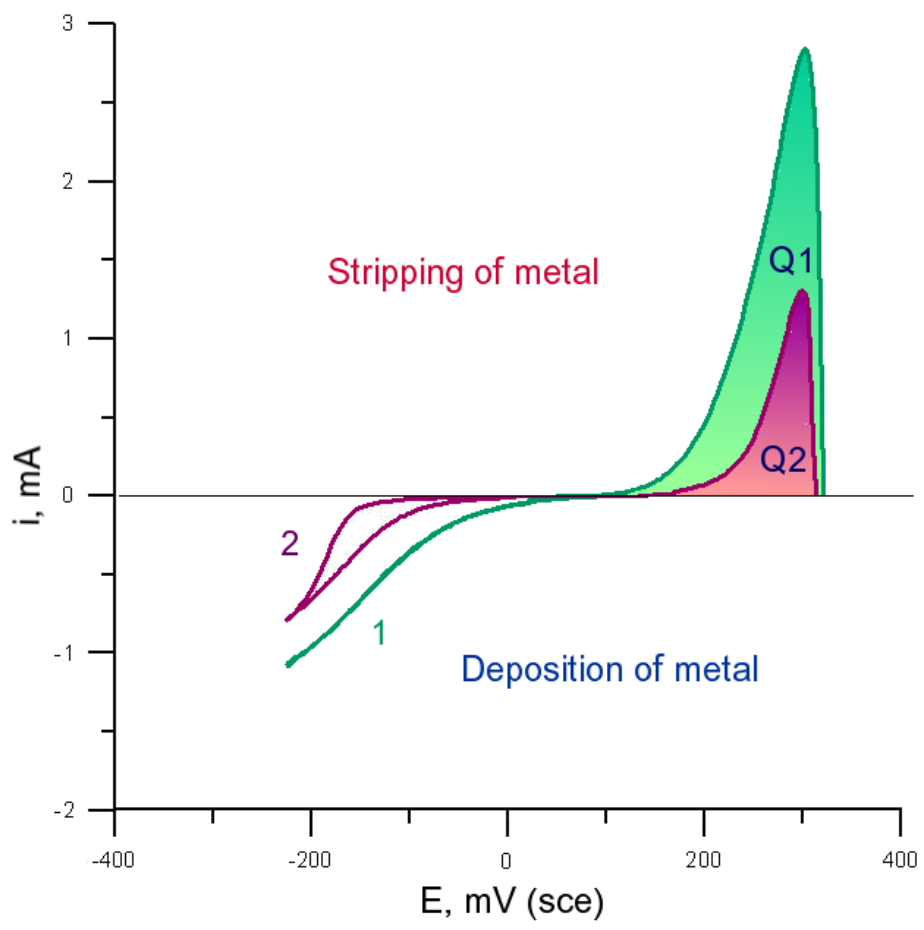

Figure 1. Voltammograms of the copper deposition and dissolution in the sulfuric acid electrolyte without additive (1) and with additive (2). 
The principle of the "Korian" method is based on CVS - curves recorded at an inert indicator electrode (platinum or glassy carbon) in the potential range, where the metal is deposited and dissolved alternately (Figure 1). The area under the peak of the deposit stripping corresponds to the charge $(Q)$ required to dissolve the metal and is proportional to the rate of its cathodic plating. This method excludes the influence of parallel cathodic reactions (reduction of hydrogen, monovalent metal ions, etc.) on the charge value $Q$. The change in the additive concentration in the electrolyte can be determined by the change in the peak area of the galvanic deposit discharge $(Q)$. If the additive inhibits the cathodic process, then the area under the peak of the anodic dissolution of the galvanic deposit $Q$ decreases (Figure 1, curve 2), if the activating additive accelerates the deposition of the metal, then $Q$ increases. Thus, a change in $Q$ will connect with a change in the concentration of organic matter in the electrolyte.

In order to minimize the effect of the change in the state of the platinum indicator electrode surface and the composition of solution, the value of the relative metal deposition rate $Q / Q_{0}$ is taken as the analytical signal, instead of $Q$. Here $Q$ is the metal deposition rate in the electrolyte containing an organic additive, and $Q_{0}$ is the deposition rate in the solution without additive (the background level).

The value of the analytical signal $Q / Q_{0}$ depends on the optimal potential ranges and the sweeping rate of the CVS (or PCVS) curves, as well as on hydrodynamic conditions at the electrode, if the cathodic process is controlled by the reducing ions diffusion. The sensitivity and reproducibility of the results also strongly depends on the pre-treatment of the indicator electrode surface [5-7]. To ensure the constancy of the hydrodynamic conditions at the indicator electrode during the analysis, a rotating micro disk electrode has been used. The pretreatment includes anodic polarization in the region of positive potentials, at which the organic matter is oxidized on the indicator electrode by the evolved oxygen. It is very important, especially for a platinum electrode.

The specific parameters of the method depend on the nature of the electrochemical system "electrolyte-organic additive", therefore, for each type of the galvanic bath (copper plating, zinc plating, tin plating, etc.), they must be determined experimentally.

Thus, the main condition for the implementation of the described analysis technique is the effect of organic matter on the metal deposition rate (inhibition or acceleration), which makes the method universal, i.e. independent of the nature of the organic additive.

For the quantitative processing of the results, special calibration methods are proposed. So, to determine inhibiting additives, the method of voltammetric titration with dilution was used. To determine the concentration of brighteners, a linear approximation method or a modified linear approximation of the coulometric dependence can be applied.

The "Korian" method has a high sensitivity $(0.01 \mathrm{ml} / \mathrm{L})$ and allows determining quickly (in 5-10 $\mathrm{min}$ ) the concentration of single- and multicomponent organic additives of various natures in various types of the galvanic bathes with an error no more than 5\%. The "Korian" method is adapted to the IPC-Compact or IPC-Pro potentiostats by means of a specialized application for a personal computer in addition to the standard program for the given device 
[8] (Figure 2). This program allows to carry out fully automate analysis of additives by controlling the operating modes of the potentiostat. Analysis results are presented in digital and graphic forms.

The "Korian" program allows not only to quantitatively assess the organic additives concentration, or effectiveness, but also to determine their optimal dosage into the galvanic bath, to carry out incoming control of consignments of additives. In addition, the "Korian" program allows evaluating the stability of additives during electroplating, as well as during storage of the electrolyte.

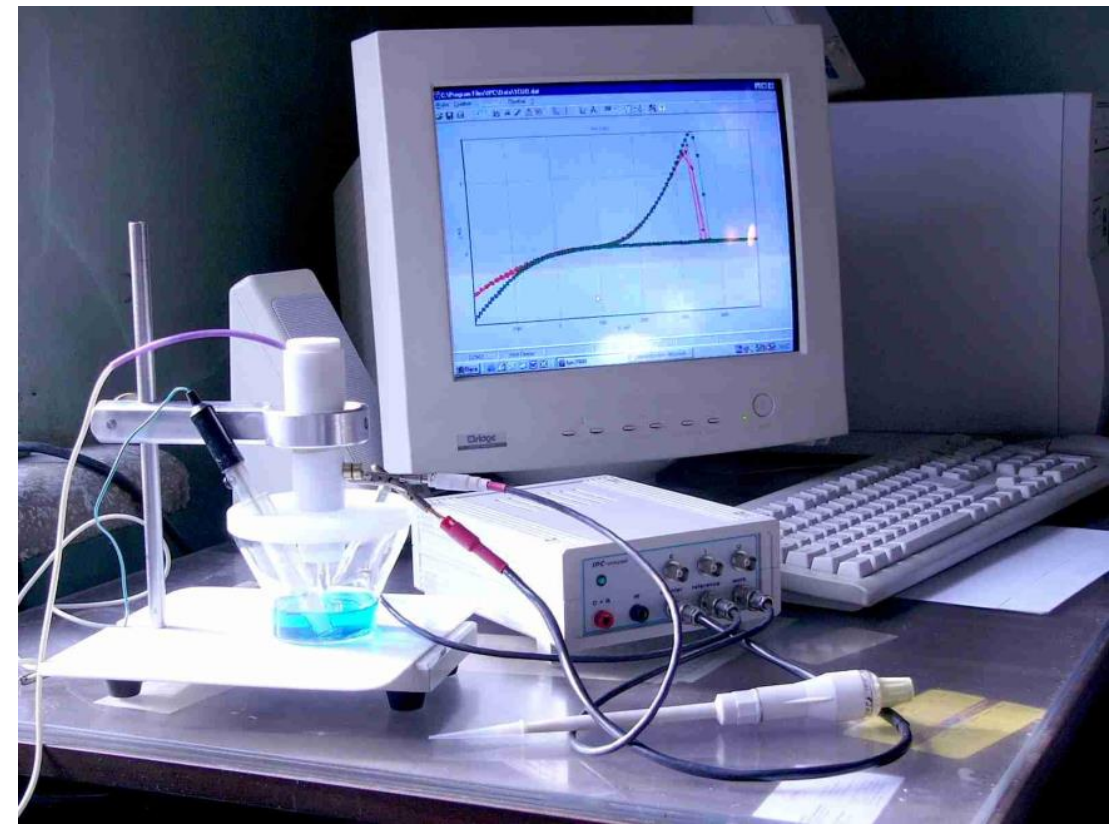

Figure 2. Hardware and software complex for the Korian method based on the IPC-Compact potentiostat.

\section{Experimental}

Almost all modern electrolytes for metal electrodeposition contain inhibitory additives. They are used to improve the throwing power of an electrolyte and to obtain uniform electrolytic deposits, which is especially important in the case of complexly shaped parts. Figure 3 shows the change in the analytical signal depending on the concentration of the inhibitor in the copper plating electrolyte when it is introduced into the background electrolyte without any additive. Even at a concentration level less than $0.01 \mathrm{ml} / \mathrm{L}$, the inhibitor affects the metal deposition rate, reducing it. As the inhibitor concentration increases, the analytical signal $Q / Q_{0}$ (and the metal deposition rate, correspondingly) drops sharply, and then reaches a constant level at the additive concentrations about $5-6 \mathrm{ml} / \mathrm{L}$, usually used in practice. This type of change in the analytical signal $Q / Q_{0}$ is the same for a number of electrolytes containing different concentrations of the inhibitor. The only difference is that the higher the inhibitor concentration in the electrolyte, the more sharply $Q / Q_{0}$ changes (compare Figure 3 curves 1 and 2). The concentration of the additive is estimated using the potentiometric 
dilution titration method, when $Q / Q_{0}=0.5$. In addition, the calculation of the inhibitor concentration can be carried out using the inflection point of the S-shaped curve.

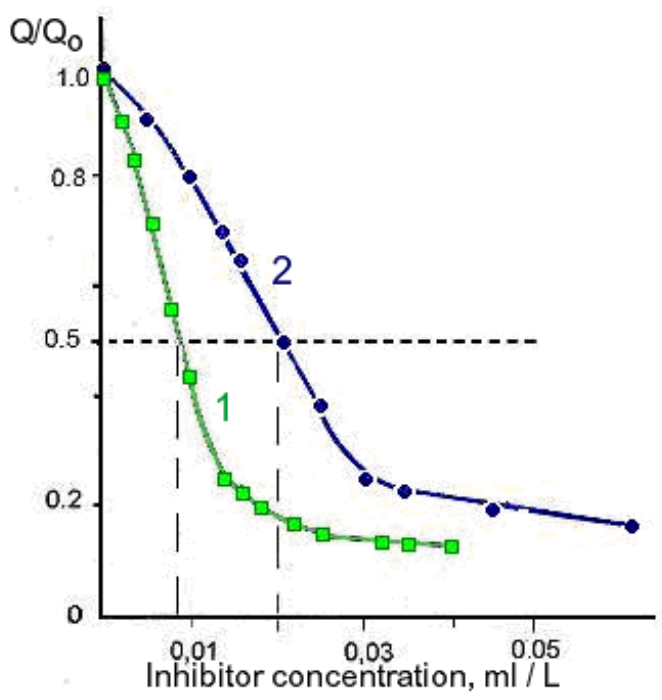

Figure 3. Dependence of the analytical signal $Q / Q_{0}$ on the inhibitor concentration in the copper plating electrolyte. 1: $10 \mathrm{ml} / \mathrm{L}$. 2: $5 \mathrm{ml} / \mathrm{L}$.

When using an inhibitor, its stability is very important. Figure 4 shows how the stability of the inhibitor changes in an idle galvanizing electrolyte (during its storage). From Figure 4 (curves 1 and 2) it is evident, that the dependence of $Q / Q_{0} v s$. the inhibitor concentration becomes less sharp with a time of storage. In terms of concentration, the content of the inhibitor decreases from 10 to $4.2 \mathrm{ml} / \mathrm{L}$. It is very important to assess in this case how much the effectiveness of the inhibitor has changed.

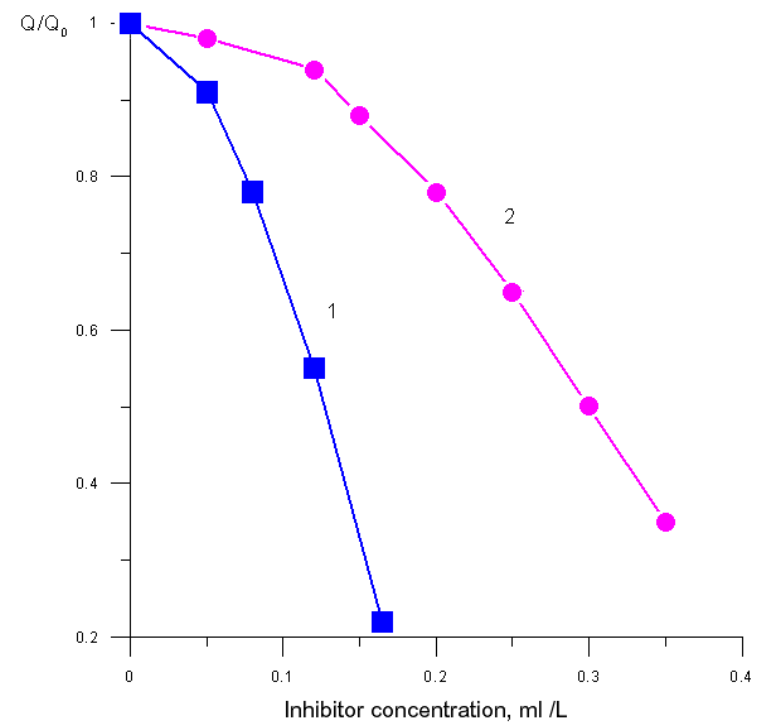

Figure 4. Change in the analytical signal $Q / Q_{0} v s$. inhibitor concentration the idle electrolyte for Zn plating. 1: after fresh addition to the bath; 2: after storage for 10 months. 
The effectiveness of the additive $(A)$ is understood as the degree of inhibition of the cathodic process under the influence of organic matter, expressed as a percentage. The value of the effectiveness of the additive is calculated by the formula:

$$
A=\left(1-Q / Q_{0}\right) \cdot 100 \%,
$$

where $Q / Q_{0}$ is the relative rate of metal deposition. It is obvious that in the electrolyte without additive $A=0$. On the contrary, with complete inhibition of the cathodic process, when the metal deposition completely stops, $A=100 \%$. Thus, parameter $A$ is a quantitative characteristic of the effect of an organic additive (or the products of its interaction with the electrolyte and/or electrodes), especially when the stability of the additive changes significantly and its consumption is high in the plating process [9]. Changes in the effectiveness of the inhibitor can be illustrated by the example of studying the stability of some additive "M", which is widely used in sulfuric acid electrolytes of copper plating for the manufacture of printed circuit boards (PCB). When stored for a long time in a closed container (1-1.5 years), the additive loses its inhibitory properties. The effectiveness of the additive falls from $95 \%$ (Figure 5, curve 1) to 35-40\% (Figure 5, curve 2). It should be noted that an increase in the concentration of the additive that has been standing for 1.5 years does not enhance its inhibitory effect, since an organic components is subject to "aging" and transformation into another ineffective substance. It turned out that, despite the fact that the quality of the coating did not deteriorate, the throwing power of the bath decreased significantly.

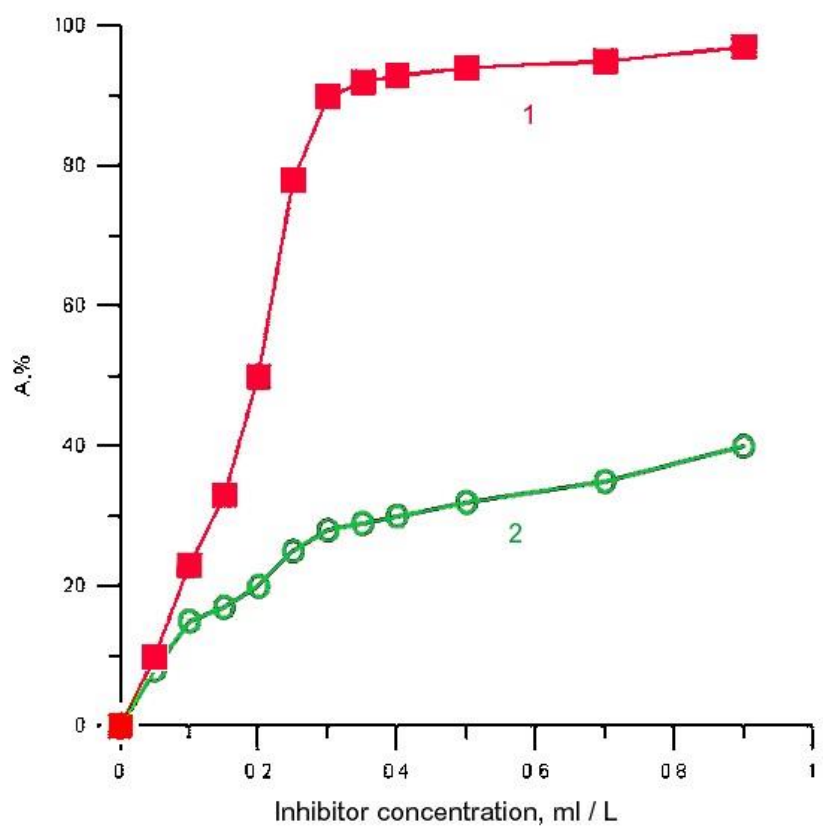

Figure 5. Dependence of the inhibitor efficiency on its concentration in the sulfuric acid electrolyte for copper plating. 1: fresh batch of inhibitor additive; 2 : the same additive after 1.5 years of storage. 
Electrolytes for metal deposition often contain not one, but several inhibitors, as well as a brightener additive, such as copper plating bathes used in the manufacture of PCB. Brighteners are introduced in dozens of times lower concentrations than inhibitors and play an important role in obtaining smooth and sometimes shiny deposits. The effect of brighteners is controlled by the adsorption process; they are included to the applied coating, changing its structure. Like inhibitors, brighteners are unstable in the electrolyte. Unlike inhibitors, the concentration of the brightener in the electrolyte does not decrease, but increases due to the formation of biproducts (oxidation or reduction of organic matter). An increase in the concentration of the brightener has a negative effect on the physical and mechanical properties of the metal. This is especially critical in the manufacture of PCB technology, when the coating literally "explodes" due to high internal stresses during soldering. In this regard, control of the brightener content in the electrolyte is especially important. In addition, the properties of the electrolytic coating can be influenced by the photoresist products accumulating in the electrolyte.

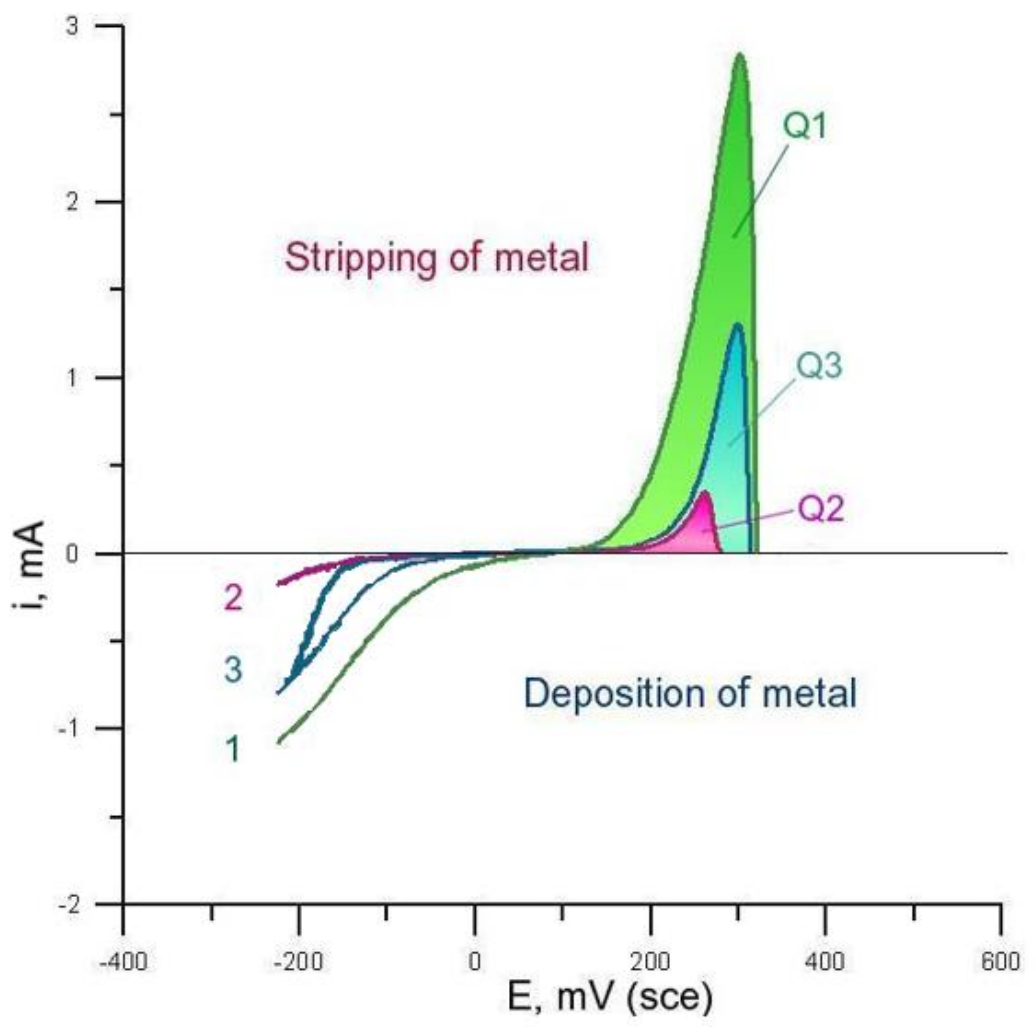

Figure 6. Voltammograms: 1 : in the electrolyte without organic matter; 2: in the electrolyte before cleaning; 3 : after treatment with hydrogen peroxide and activated carbon.

As you know, copper plating electrolytes used in the production of printed circuit boards are periodically cleaned from organic impurities. For this purpose, organic substances are oxidized with hydrogen peroxide, followed by treatment with activated carbon. Cyclic voltammetry with stripping of the metal makes it possible to assess the degree of electrolyte purification from organic matter. Comparison of the dissolution peaks of copper deposits 
from the indicator electrode in an electrolyte without organic matter (Figure 6, curve 1), in an electrolyte with organic impurities before cleaning (Figure 6, curve 3) and after cleaning (Figure 6, curve 2) shows how thoroughly the electrolyte was purified from impurities. The more the dissolution peaks of the precipitate in the electrolyte with impurities differ from the electrolyte without organic matter, the worse the electrolyte is purified.

Thus, from the presented examples of the "Korian" technique application, the broad possibilities of the method are visible. The employment of the "IPC-KORIAN" hardwaresoftware complex allows to obtain fully automate analysis of organic additives in the electroplating bathes. This device is easy to operate and has found application in dozens of plants in Russia and abroad.

\section{References}

1. E. Eger and A. Zalkind, Measurement methods in electrochemistry, 1977, Moscow, Nauka, 1, 588; 2, 476 (in Russian).

2. W. Nohse, The Hull Cell, 1966, Teddington, R. Draper Ltd., 122.

3. K.N. Smirnov, Some practical recommendations for the operation of nickel-plating electrolytes, Gal'vanotekh. Obrab. Poverkhn., 2005, XIII, no. 2, 6-9 (in Russian).

4. S.S. Kruglikov, N.T. Kudriavtsev, G.F. Vorobiova and A.Ya. Antonov, On the mechanism of leveling by addition agents in electrodeposition of metals, Electrochim. Acta, 1965, 10, 253-261. doi: 10.1016/0013-4686(65)87023-2

5. L.N. Solodkova, V.N. Kudryavtsev and Z.A. Solovieva, USSR Patent No. 1686927, 22.06.1991 (in Russian).

6. L.N. Solodkova, Cyclic voltammetry (CVS) as a method for determining the concentration of organic additives in electrolytes for metal electrodeposition, Collection of abstracts of reports at the International Conference dedicated to the 200th anniversary of B.S. Jacobi "Electrochemistry, electroplating and surface treatment", Moscow, 4-8 June 2001, 109 (in Russian).

7. L.N. Solodkova and V.N. Kudriavtsev, A Method for Determining Additive Concentration in Alkaline Non cyanide Zinc Plating Baths, Met. Finish., 1996, 94, no. 5, 26-31. doi: 10.1016/S0026-0576(96)80006-7

8. V.E. Kasatkin, L.N. Solodkova and Yu.V. Kondrashov, Potentiostats of IPC series: application practice in electrochemical research methods. Part 1. Analyzer of organic additives "Corian-3", Gal'vanotekh. Obrab. Poverkhn., 2011, XIX, no. 2, 27-34 (in Russian).

9. L.N. Solodkova, Evaluation of the effectiveness of organic additives in electrolytes for metal electrodeposition, Collection of abstracts of reports at the Annual All-Russian scientific-practical conference "Electroplating, surface treatment and ecology of the XXI century”, 2003, Moscow, April, 119-121 (in Russian). 